\title{
THE CHARACTERISTICS OF THE MICROCOLONIES OF SOME PATHOGENIC COCCI*
}

\author{
Reba Cordelia Haner and William Dodge Frost \\ From the Agricultural Bacteriological Laboratories, University of Wisconsin, Madison
}

A new method for the study of bacteria was recently described by one of us, ${ }^{1}$ in which it was pointed out that it was possible to dry down and stain colonies of bacteria only a few hours old. In these microcolonies the cell form and structure are quite as well brought out as they would be in a cover glass preparation and in addition the relation of cell to cell is easily studied. These colonies appear to show distinctive characters and the method promises to be of material aid in the study and recognition of bacteria.

It is proposed in this paper to describe the results of a study of the microcolonies of some staphylococci, streptococci and pneumococci.

\section{METHOD}

The culture medium for the staphylococci was sterile milk and $1 \%$ nutrient agar (dehydrated Difco) equal parts. For the streptococci and pneumococci, $0.5 \mathrm{cc}$ horse or rabbit serum or whole blood was added to a tube of the milk and agar mixture (6 to $12 \%$ ). The organisms were all given a preliminary culture in a liquid medium for a few hours.

The "little plates" were made by placing two small drops of the liquefied medium on a glass slide which had just been sterilized in the flame. Into one drop a loopful of the preliminary culture was put and spread about into a thin film. The loop without resterilization was then used to spread the second drop. In this way two "little plates" were formed, one of which was a dilution of the other. They were then incubated in a moist chamber for 4 hours. The form of moist chamber is immaterial. For a few slides, Petri dishes may be used. In this case a piece of wet filter paper is placed in the bottom and the slides rested on glass rods or match sticks. A convenient form is a "moist chamber cabinet" designed especially for the purpose (Central Scientific Company of Chicago). An incubation period of from 4 to 6

Received for publication, Dec. 8, 1920.

I Frost, Jour. Am. Med. Assn., 1919, 72, p. 323. 
hours seems best. Younger colonies are likely not to be characteristicolder ones are frequently too dense to reveal the structure. When removed from the moist chamber they were dried down very rapidly on a steam plate and then put in a stain made thus: thionine blue, $1 \mathrm{gm}$.; phenol, $2.5 \mathrm{gm}$. ; distilled water, $400 \mathrm{gm}$.; and glacial acetic acid, $20 \mathrm{c} \mathrm{c}$.

Two minutes was sufficient, but a longer time does not cause overstaining. The slides were thoroughly washed in tap water and dried either at room temperature or on the steam plate. They were examined under the immersion objective by putting the oil directly on the preparation.

The photomicrographs of the colonies were made by means of a horizontal camera suspended on springs. The source of light was 1,000 watts mazda with condensed filament. The Wratten and Wainwright "M" Plates together with the Wratten filter B and $E$ were used.

The following cultures were studied:

(a) Staphylococcus pyogenes var. aureus, one strain.

Staphylococcus pyogenes var. albus, one strain.

(b) Hemolytic Streptococci

Streptococcus pyogenes, one strain.

Streptococcus epidemicus, one strain.

Streptococcus alactosus, one strain.

Streptococcus hemolyticus, none.

(c) Nonhemolytic Streptococci

Strain mitis, 11 strains.

Strain fecalis, 3 strains.

Strain salivarius, 2 strains.

Strain unidentified, 9 strains.

(d) Pneumococci

Type 1,9 strains.

Type 2, 9 strains.

Type 3, 10 strains.

Type 4, 3 strains.

\section{MICROCOLONY CHARACTERISTICS OF THE STAPHYLOCOCCUS GENUS}

The microcolonies of the staphyloccus genus are of medium size, with a more or less dense, compact center. At the edge of the colony are seen flattened or biscuit-shaped diplococci, frequently in a tetrad 
grouping. The mode of division of the cell seems to be in two planes, i. e., at right angles to each other.

The albus colony seems to be more open and shows the typical tetrad grouping more plainly than the aureus, which, on the other hand, has a dense center with a few biscuit-shaped diplococci, often in tetrads, on the border of the colony.

\section{MICROCOLONY CHARACTERISTICS OF THE STREPTOCOCCUS GENUS}

The streptococcus genus is usually divided into two groups, the hemolytic and the nonhemolytic. Ruediger ${ }^{2}$ and afterward Holman ${ }^{3}$ have noted some morphologic differences. The hemolytic group, he says, is characterized by the formation of closely packed, frequently disk-shaped cocci in chains which have the appearance of having been compressed at right angles to the axis of the chains. On the other hand, it was noted that the nonhemolytic group is characterized by the appearance of elongated clearly marked pairs, giving the chains the appearance of having been stretched.

In agreement with Ruediger and Holman, these general differences have been observed in the study of a number of strains and are obvious in the photomicrographs.

The microcolonies of the hemolytic group show the single cocci in chains. The individuals are frequently not spherical, but rather compressed at right angles to the axis of the chains. The closely packed, frequently disk-shaped, individual cocci give them a tightly bound, firm appearance. Some microcolonies of the hemolytic group show a somewhat dense center with some disk-shaped or spherical diplococci at the outer edge of the colony. The colonies of this type have no chains, but the cocci at the border of these colonies are quite noticeably spherical and occur in pairs. The microcolonies are large, some are circular in form-especially the compact colony-while others are angular in form and seem to spread in various directions with the interlacing of the chains.

The microcolonies of the nonhemolytic group are characterized by the elongated diplococci which are distinctly separated from the next pair, but are joined together in a loose chain. The separation of the

2 Jour. Infect. Dis. ,1906, 3, p. 761.

s Jour. Med. Research, 1915, 34, p. 392. 
diplococci gives the chains the appearance of having been stretched. Some microcolonies of this group show a rather dense center with a few elongated diplococci at the edge of the colony, with no tendency to form chains.

The microcolonies of this group are also large; some are angular with the interlacing of chains, while others are somewhat circular with the dense center and no chains. Colonies of this type are difficult to distinguish from the microcolonies of the dense type of the hemolytic group.

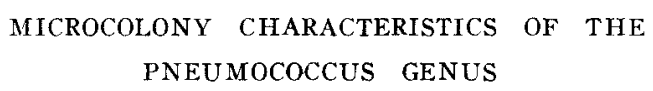

The microcolonies of the pnetmococcus genus are small and open with the cells arranged in a free and distributed manner. The lancetshaped cells are grouped together as diplococci. There is a slight tendency toward chain formation in type 3 , which by some is called Streptococcus mucosus and in type 4 which includes various unknown pneumococci.

Contrasting the pneumococcus microcolonies with those of the streptococci: In general the pneumococcus colonies are very small, composed of lancet-shaped diplococci, which may occur in short chains or alone. The small size of the colony is one of the noticeable characteristics. The streptococcus microcolonies are considerably larger, with the diplococci arranged in closely packed chains in some strains or in loosely connected chains in others.

\section{DOES ANimal PASSAGE influtence the CHARACTER OF THE COLONY?}

In the study of the pneumococcus, the question was raised: Is the colony formation altered by animal passage or, in other words, would the colonies be different if the organisms came directly from a diseased condition? In order to study this, white mice were inoculated intraperitoneally and the organism recovered from the heart blood. Cultures of several strains on blood agar were used, and "little plates" of the organism from the heart blood were grown and their morphologic characteristics determined. The character of the microcolony does not seem to be altered by animal passage, except that in a few cases the colonies are somewhat smaller than the microcolonies of the same organism before animal passage. 


\section{Explanation of Plate I}

Colonies of Staphylococci 5 Hours Old Incubated at $37 \frac{1 / 2}{\mathrm{C} \text {. }}$

Fig. 1.-Colony of Staph. pyogenes, var. albus.

Fig. 2.-Colony of Staph. pyogenes, var. aureus.

Colonies of Nonhemolytic Streptococci 4 Hours Old Incubated at $371 / 2 \mathrm{C}$.

Fig. 3.-Colony of Strep. mitis.

Fig. 4.-Colony of Strep. mitis.

Fig. 5.-Colony of Strep. fecalis.

Fig. 6.-Colony of Strep. salvarius.

Fig. 7.- Colony of strep. from case of ozena.

Fig. 8.-Colony of strep. from case of scarlatina.

Fig. 9.-Colony of strep. from case of acute articular rheumatism. Colonies of Hemolytic Streptococei 4 Hours Old Incubated at $37 \% / 2 \mathrm{C}$.

Fig. 10.-Colony of Strep. pyogenes.

Fig. 11.-Colony of Strep. epidemicus.

Fig. 12.-Colony of Strep. alactosus.

Fig. 13.-Colony of a hemolyite streptococcus.

Fig. 14.- Same organism as in fig. 13, but a deep colony.

Fig. 15.-Colony of a hemolytic streptococcus.

Fig. 16.-Colony of a hemolytic streptococcus from case of otitis.

Fig. 17.-Deep colony of a hemolytic streptococcus from case of mastitis.

Fig. 18.-Same organism as in fig. 17 but a surface colony. 
Plate 1
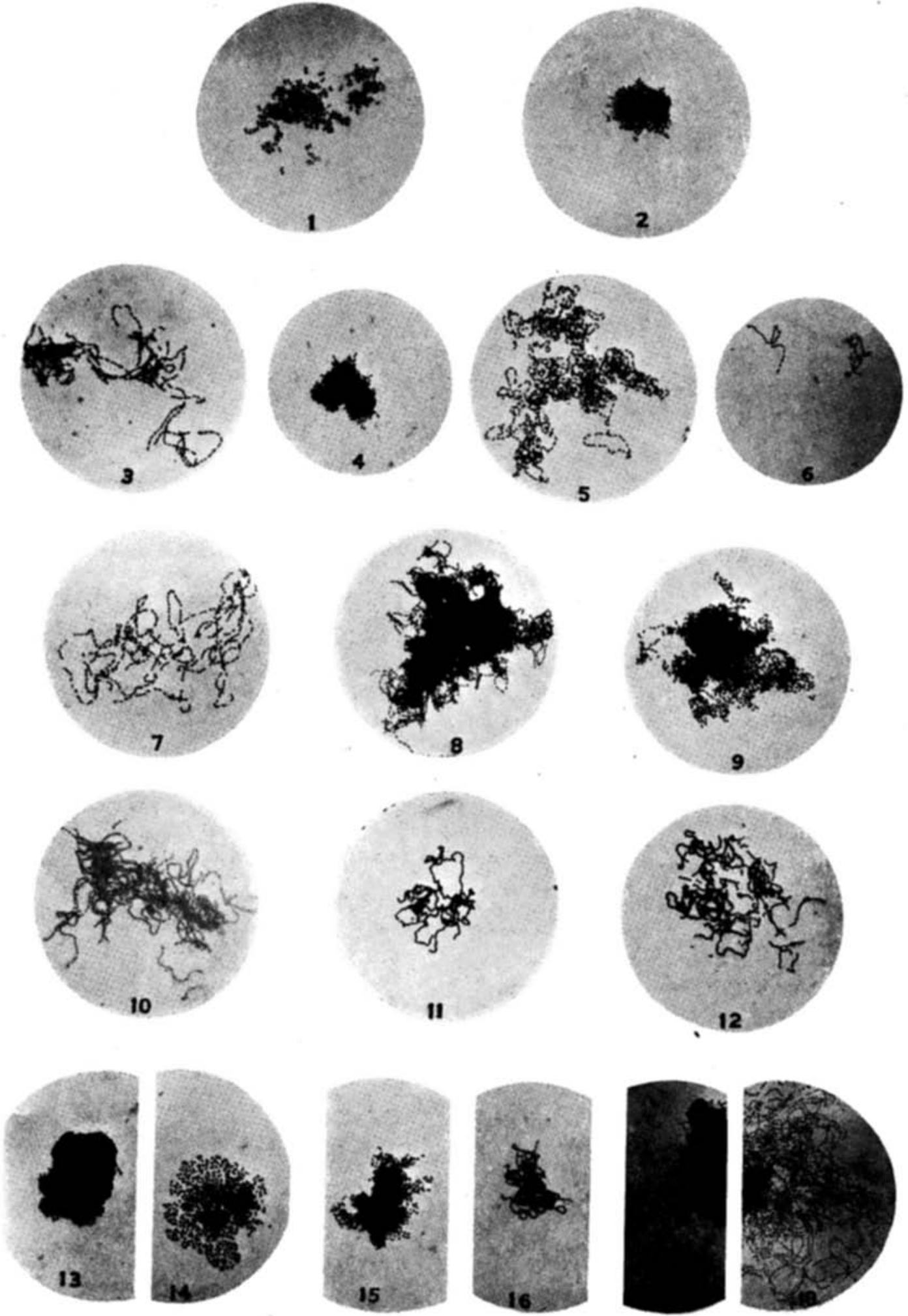


\section{Explanation of Plate II}

Colonies of Pneumococci Type 1, 4 Hours Old Incubated at $37 \frac{1}{2} \mathrm{C}$.

Fig. 1.-Colony of pneumococcus type 1.

Fig. 2.-Colony of pneumococcus type 1.

Fig. 3.-Colony of pneumococcus type 1 from heart blood of mouse. Culture same as in fig. 2 .

Colonies of pneumococei type 2, 4 Hours Old Incubated at $37 \frac{1}{2} \mathrm{C}$.

Fig. 4.-Colony of pneumococcus type 2.

Fig. 5.-Colony of pneumococcus type 2 from heart blood of mouse; culture same as in fig. 4 .

Fig. 6.-Colony of pneumococcus type 2 .

Fig. 7.-Colony of pneumococcus type 2 from heart blood of mouse; culture same as used in fig. 6 .

Colonies of pneumococcus type 3,4 Hours Old Incubated at $371 / 2 \mathrm{C}$.

Fig. 8.-Colony of pneumococcus type 3 .

Fig. 9.-Colony of pneumococcus type 3 from heart blood of mouse; same strain as in fig. 8 .

Fig. 10.-Colony of pneumococcus type 3 .

Fig. 11.-Colony of pneumococcus type 3 from heart blood of mouse; culture same as in fig. 10 .

Fig. 12.-Colony of pneumococcus type 3.

Fig. 13.-Colony of pneumococcus type 3 from heart blood of mouse; culture same as in fig. 12 .

Fig. 14.-Colony of pneumococcus type 3 from heart blood of mouse 2nd passage; culture same as in fig. 12 .

Colonies of pneumococcus type 4, 4 Hours Old Incubated at $37 \frac{1}{2} \mathrm{C}$.

Fig. 15.--Colony of pneumococcus type 4 .

Fig. 16. - Colony of pneumococcus type 4 from heart blood of mouse; culture same as in fig. 15. 

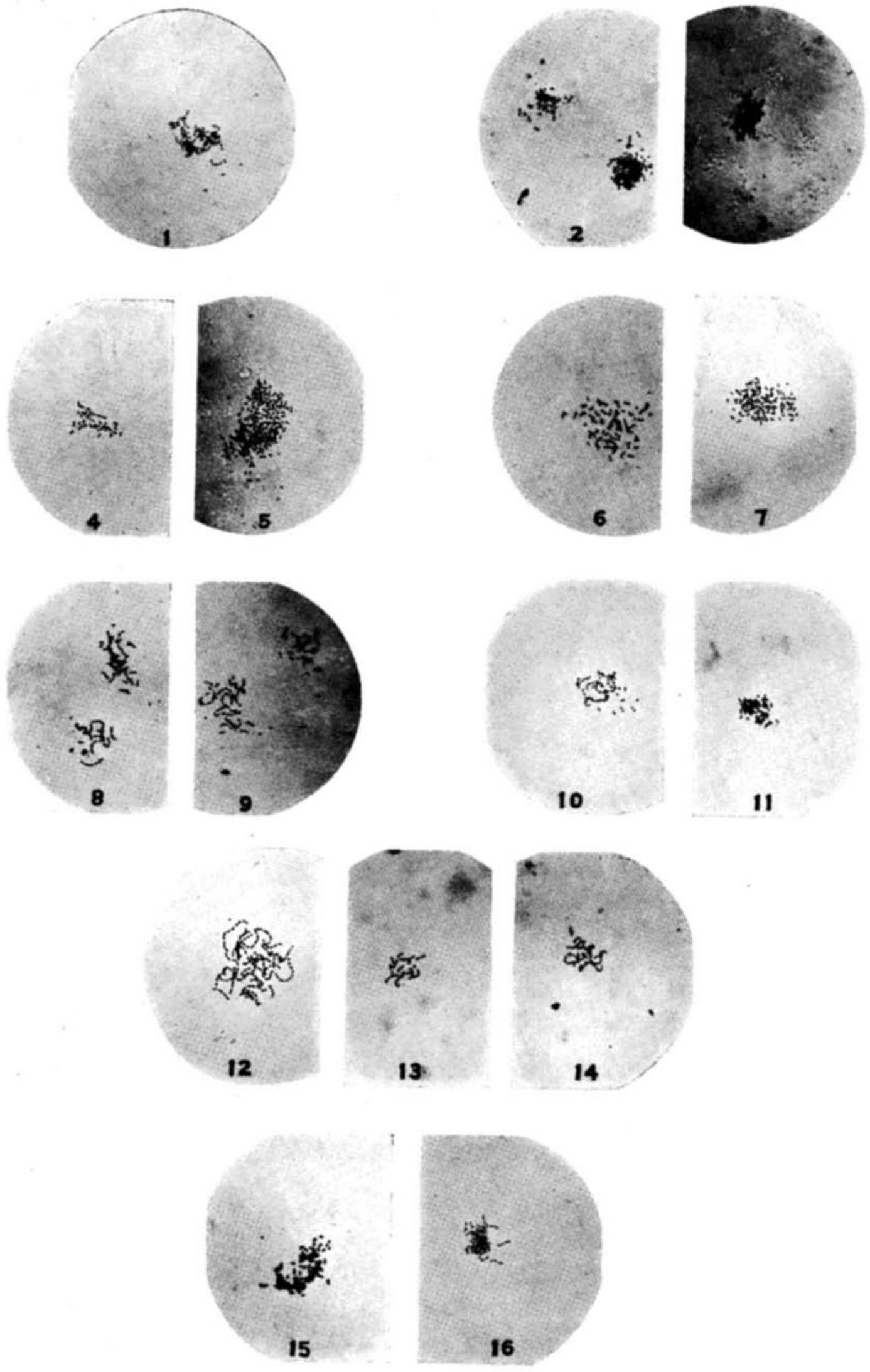